\title{
Ictiobot-40 a low cost AUV platform for acoustic imaging surveying
}

\author{
Gerardo G. Acosta \\ INTELYMEC-CIFICEN \\ (UNCPBA, CICPBA, CONICET) \\ FIO, UNCPBA \\ Olavarría, Argentina \\ ggacosta@fio.unicen.edu.ar
}

\author{
Bruno V. Menna \\ INTELYMEC-CIFICEN \\ (UNCPBA, CICPBA, CONICET) \\ FIO, UNCPBA \\ Olavarría, Argentina \\ bruno.menna@fio.unicen.edu.ar
}

\author{
Ignacio Carlucho \\ INTELYMEC-CIFICEN \\ (UNCPBA, CICPBA, CONICET) \\ FIO, UNCPBA \\ Olavarría, Argentina \\ ignacio.carlucho@fio.unicen.edu.ar
}

\author{
Mariano De Paula \\ INTELYMEC-CIFICEN \\ (UNCPBA, CICPBA, CONICET) \\ FIO, UNCPBA \\ Olavarría, Argentina \\ mariano.depaula@fio.unicen.edu.ar
}

\author{
Sebastian A. Villar \\ INTELYMEC-CIFICEN \\ (UNCPBA, CICPBA, CONICET) \\ FIO, UNCPBA \\ Olavarría, Argentina \\ svillar@fio.unicen.edu.ar
}

\author{
Hugo J. Curti \\ INTELYMEC-CIFICEN \\ (UNCPBA, CICPBA, CONICET) \\ FIO, UNCPBA \\ Olavarría, Argentina \\ hcurti@fio.unicen.edu.ar
}

\author{
Alejandro F. Rozenfeld \\ INTELYMEC-CIFICEN \\ (UNCPBA, CICPBA, CONICET) \\ FIO, UNCPBA \\ Olavarría, Argentina \\ alex@fio.unicen.edu.ar
}

\author{
Roberto J. de la Vega \\ Electromechanical Engineering \\ Department \\ FIO, UNCPBA \\ Olavarría, Argentina \\ rjdlv@fio.unicen.edu.ar
}

\author{
Agustin Isasmendi \\ Electromechanical Engineering \\ Department \\ FIO, UNCPBA \\ Olavarría, Argentina \\ aisasmendi@fio.unicen.edu.ar
}

\author{
Roberto C.Leegstra \\ Electromechanical Engineering \\ Department \\ FIO, UNCPBA \\ Olavarría, Argentina \\ rleegstra@fio.unicen.edu.ar
}

\author{
Luis M. Arrien \\ Electromechanical Engineering \\ Department \\ FIO, UNCPBA \\ Olavarría, Argentina \\ lmarrien@fio.unicen.edu.ar
}

\begin{abstract}
Autonomous Underwater Vehicles (AUVs) are suitable platforms for a wide type of applications in the oceanic environment. These applications are developed in various fields such as scientific surveying, off-shore industry and defense. The employment of AUVs requires less human support and reduces operation costs. Due to the changing marine environment these vehicles must deal with uncertain and hostile conditions to perform its tasks. In the marine robotics matter, the INTELYMEC group has developed in 2012 an AUV prototype called Ictiobot, a low cost experimental platform for multipurpose missions. In this paper an upgrade of the original prototype is presented, the Ictiobot-40, conceived to perform acoustic imaging surveying missions of up to two hours and maximum depths of 40 meters. The new software and hardware architectures and mechanical structure improvements, are detailed. In addition to these technical details, initial experimental results of the AUV performance in quiet waters will be discussed. Also, the new approaches for systems under development are presented.
\end{abstract}

Keywords-Autonomous Underwater Vehicle, acoustic surveying, Local Interconnect Network, Robot Operating System, SONAR

\section{INTRODUCTION}

Since its early developments, the continuous advance in Autonomous Underwater Vehicles (AUV) technology has allowed its active employment in a wide type of tasks in the oceanic environment. These platforms may carry a diverse sensor payload, which combined with the vehicle mobility and autonomy allow to make both spatial and time series measurements. In addition, due to the less user intervention, operational costs are reduced [1] and [2]. The fields in which AUVs are used include; hydrographic and scientific survey, offshore industry and defense [3], [4], [5] [6] [7] and [8]. Due to the extreme nature of the underwater environment, that includes high pressures, corrosive effects of seawater and the possibility of damage by bad sea state or collisions, the reliability of AUVs is a topic of interest to avoid the loss of high cost equipment [9].

As a result of the increasing use of AUVs, payload and computational capabilities requirements have grown. In addition, versatility and low cost are also important. In this sense INTELYMEC group has carried out several research and developments [10] and [11]. The most recent development of the group is a complete upgrade of an earlier project of an AUV prototype named Ictiobot. The new version, the Ictiobot-40, consists of an AUV with a maximum depth rate of 40 meters and with a payload for acoustic imaging surveying missions.

In the Ictiobot-40, a new electronic architecture was implemented, consisting in a network of microcontroller nodes to perform low level sensing, actuating and fault detection tasks. Software architecture was also improved based on the Robot Operating System [12] and [13]. These improvements allowed to achieve a more reliable platform that maintains the premises of low cost, simplicity, ease of transport and deployment and flexibility to continue with other research works of the group.

This paper is organized as follow; in Section II the general architecture of the Ictiobot 40 is described and the mechanical, 
electronic, payload and software designs are detailed. In Section III the mission planning, main systems and its interactions are presented in conjunction with the initial test results. In Section IV conclusions are discussed from which the future works are considered to complete the Ictiobot 40 development.

\section{ICTIOBOT40 GENERAL ARCHITECTURE}

Ictiobot-40 is a low cost AUV conceived to perform acoustic image surveying using a Mechanical Scanning SONAR (MSS) at depths up to 40 meters. Autonomy is limited to two hours. It's also equipped with a Forward Looking SONAR (FLS) for obstacle detection. Given the thrusters configuration, Ictiobot40 may perform surge, heave, yaw and pitch movements. In addition to operating autonomously, the Ictiobot-40 can be remotely operated in surface from a PC through an UHF (Ultra High Frequency) link or via an Ethernet WiFi connection.

The general architecture of the Ictiobot-40 may be described as a set of systems implemented in software and hardware components. In Fig. 1 a simplified diagram of the general architecture the Ictiobot-40 is shown, this diagram shows the main systems and hardware components and the interactions between them.

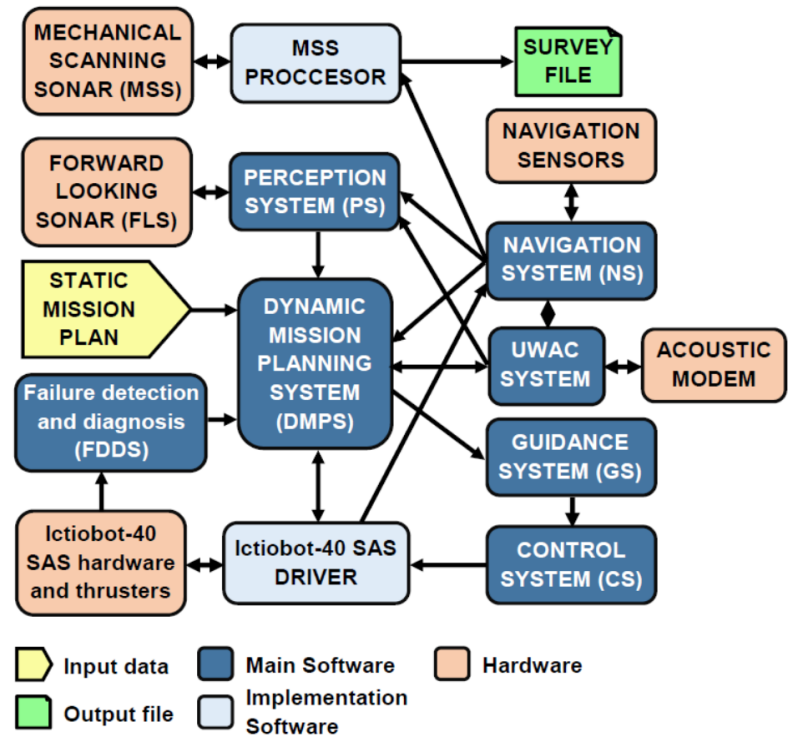

Fig. 1. Ictiobot-40 general architecture.

A brief description of the hardware and software systems is described in the next subsections.

\section{A. Main software blocks}

Main software blocks refer to the elemental systems that an autonomous vehicle must have to operate in a certain environment.

- Static Mission Plan (SMP): are the long term objectives of the mission set by the user and are considered as stable objectives that don't change in the short term. In the case of the Ictiobot-40 the static mission plan consists in a list of waypoints that the trajectory of the AUV must meet during the mission.
- Dynamic Mission Planning System (DMPS): generates short-term mission objectives that allows to replan the mission in response to events that may alter the normal development of the mission, without modifying the static mission plan. In the case of the Ictiobot-40 the DMPS takes the static mission plan and data from the PS to generate intermedia waypoints which position depends if there are obstacles in the trajectory. Taking data from the hardware status, the DMPS may also determine if it is possible to complete the mission with the remaining energy or suspend it and guide the AUV to a homing position, for example.

- Navigation System (NS): comprises the sensor fusion algorithms to determine the position, velocity and attitude of the AUV from the navigation sensors measurements.

- Guidance System (GS): generates the trajectories that the AUV must follow in order to accomplish the planned mission.

- Control System (CS): consists of the feedback loops that allows the AUV to comply with the velocity and attitude set points generated by the GS, generating the control signals to the propulsion system of the AUV.

- Perception System (PS): receives data from sensors and processes them to extract features of the operation environment. It provides the necessary information for the Dynamic Mission Planning System (DMPS).

- Failure Detection and Diagnosis System (FDDS): monitors the state of the vehicle's hardware to detect and identify failures that might compromise the development of the mission.

- Communication System: provides communication between the vehicle and other vehicles or remote stations through the most reliable or available channel.

\section{B. Implementation software blocks}

These software blocks depend directly of the vehicle implementation and comprises the interface to the vehicle hardware and also to the payload.

- Ictiobot-40 SAS driver: is an interface between the Ictiobot40 Sensing and Actuating System (SAS) hardware and software. This driver publishes the status of the hardware and communications as well as sensor data. It also receives the thrusters set points from the CS to command the thrusters drivers and commands from the DMPS.

- MSS processor: combines a driver for a Mechanical Scanning SONAR (MSS) and the processing steps necessaries to achieve the seabed bottom detection, this information and navigation data are stored in a survey file for post-mission processing.

\section{Hardware}

The hardware components associated to the systems of the vehicle. For the Ictiobot-40 these are: the SAS hardware, navigation instruments as Global Positioning System (GPS) receiver, Inertial Measurement Unit (IMU), Doppler Velocity Log (DVL), depth meter and range based positioning system, a Forward Looking SONAR (FLS) as perception sensor, acoustic 
modem as communication device and the Mechanical Scanning SONAR (MSS) as payload.

In the next sections the mechanical, electronics and software designs are detailed. A brief description of the payload is also provided.

\section{MECHANICAL DESIGN}

The mechanical structure of the AUV Ictiobot-40 consists of a reinforced fiberglass main hull to contain the electronics and batteries. The main hull has an aluminum cap in which connectors are mounted, the sealing is provided by two O-rings, the electronics chassis is attached to the main hull cap, thus easing its disassembly. The depth meter is contained in a smaller aluminum hull. Both hulls were tested in a pressure chamber to ensure their mechanical resistance and water tightness.

The Ictiobot-40 chassis is made out of aluminum and provides mechanical support for the main hull, thrusters, payload quill and accessories. Some details about the mechanical construction of the Ictiobot-40 are shown in Fig. 2 and 3 .
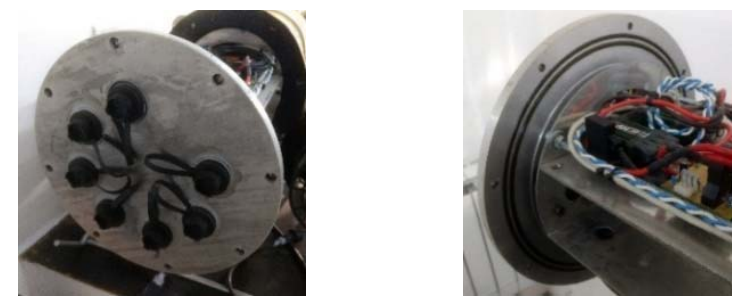

Fig. 2. Ictiobot-40 main hull end cap.

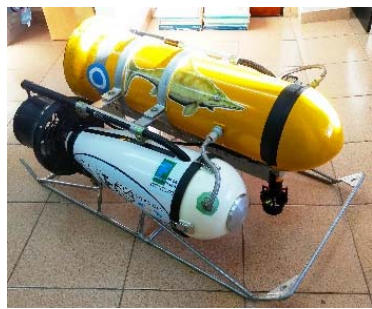

Fig. 3. Ictiobot-40 general view at the lab.

The propulsion is provided by two Torpedo 2000 thrusters at port and starboard for surge and yaw movements these thrusters have a maximum depth rate of 60 meters and also contain their own battery. The thrusters were also modified to fit a DC motor driver board with the heat sink exposed to the exterior and the electrical connection to the main hull is made via a water tight hose. Propulsion system has also two CrustCrawler ${ }^{\circledR}$ 400HFS-L brushless thrusters at bow and stern for heave and pitch movements.

During the development of the mechanical structure of the AUV a dynamic model was also obtained using CFD and CAD techniques with simulation proposes [14].

\section{ELECTRONICS DESIGN}

The electronics architecture was divided in two levels; the low level and high level electronics.
The high level electronics consists basically in a $\mathrm{PC}$ to which the navigation, perception and payload sensors are connected. The previously described software systems are implemented in this PC.

The low level is called here the Sensing and Actuating System (SAS). It has the main functions of: a) implementing a basic FDDS; b) providing sensor measurements for the NS; and c) providing the control signals for the thrusters drivers. The SAS is connected to the high level PC through an USB port.

A detailed scheme of the Ictiobot-40 electronics architecture is shown in Fig. 4.

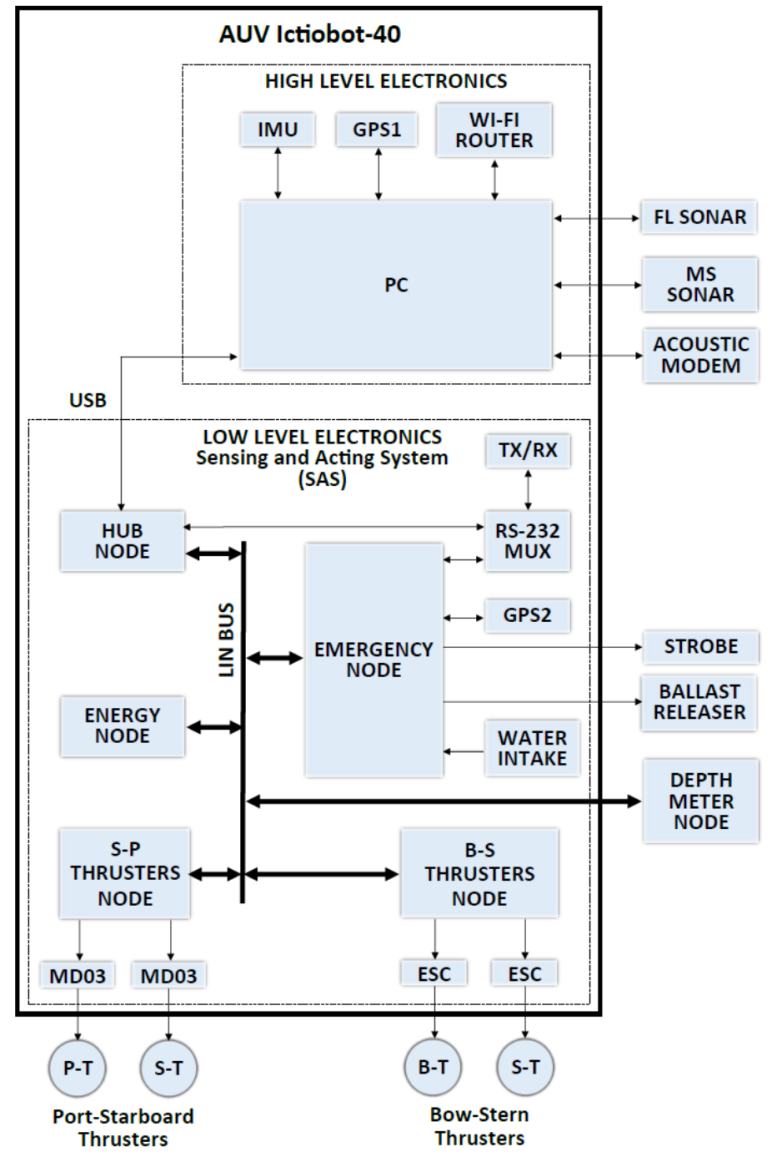

Fig. 4. Ictiobot-40 electronics architecture.

The SAS is implemented as a set of microcontroller based nodes interconnected via Local Interconnect Network (LIN) bus. Among others automotive networks like CAN, TTCAN and FlexRay, LIN is considered the most simple and inexpensive fieldbus technology. With a maximum data transfer rate of $20 \mathrm{kbps}$ and up to 16 nodes, using a single wire for transmitting data, it has a single master node which coordinates timing across the network of slave nodes, so all messages are considered to be deterministic with static latencies [15]. Current LIN Specification 2.2A is being standardized by ISO 17987 Part 1-7 [16].

The SAS nodes are implemented as a base MPS430F247 microcontroller board and an application board attached in a shield style form according to the node function. The SAS 
currently includes the energy, port-starboard thrusters and bowstern thrusters drivers interface, depth meter, emergency and hub nodes.

The energy node takes measurements of both current and voltage of the Ictiobot-40 batteries to calculate the remaining energy.

The port-starboard and bow-stern thrusters nodes receive the thrusters speed set-points and generate the control signals for the thrusters drivers. The port-starboard thrusters are driven by two MD03 DC motor drivers that are controlled with an $0-5 \mathrm{~V}$ analogic signal while the bow-stern thrusters are driven by two 50A Electric Speed Controller (ESC) that are controlled with a Pulse Wide Modulation (PWM) signal.

The depth meter node estimates the depth from the conditioned measurements of a pressure transducer.

The emergency node has water intake sensors, drives the ballast release mechanism and a strobe light. It is also connected to a GPS receiver (GPS2) and to a RS-232 multiplexer (MUX) for beaconing GPS position through the UHF transceiver in the case of an emergency situation.

The hub node, as the master node of the LIN network, interacts with other nodes in the LIN network and the high level electronics. If some SAS nodes are not responding properly or communication with the high level PC fails, the hub node detects this situation and acts consequently. This is a basic hardware implementation of the FDDS. It is based on a sequential state machine categorizing the failure degree into three meta states: NORMAL, MALFUNCTION and FAILURE. Hub node is also connected via a RS-232 multiplexer (MUX) to the UHF transceiver for sending data frames to a remote station and, once emerged allowing remote operation of the AUV.

Within each meta state there are different operation states. They are; STANDBY, REMOTE CONTROL STANDBY, ACTIVE, REMOTE CONTROL ACTIVE, SURFACING, BEACON, REMOTE CONTROL BEACON and EMERGENCY, as shown Fig. 5.

Failure conditions of the SAS nodes were considered and for every case an action in both the SAS and high level electronics was proposed. These actions are listed in Table I.

TABLE I. SAS AND HIGH LEVEL ACTIONS FOR SAS NODES FAILURES

\begin{tabular}{|c|l|l|}
\hline $\begin{array}{c}\text { SAS node } \\
\text { failure }\end{array}$ & \multicolumn{1}{|c|}{ SAS action } & \multicolumn{1}{c|}{ High level action } \\
\hline ENERGY & None & $\begin{array}{l}\text { Mission replan according to last } \\
\text { remaining energy estimation }\end{array}$ \\
\hline $\begin{array}{c}\text { DEPTH } \\
\text { METER }\end{array}$ & None & $\begin{array}{l}\text { Emerge controlled and surface } \\
\text { navigation to the homing position }\end{array}$ \\
\hline $\begin{array}{c}\text { STARBOARD } \\
\text { PORT } \\
\text { THRUSTERS }\end{array}$ & None & $\begin{array}{l}\text { Emerge controlled and switch SAS } \\
\text { to EMERGENCY state once in the } \\
\text { surface }\end{array}$ \\
\hline $\begin{array}{c}\text { BOW-STERN } \\
\text { THRUSTERS }\end{array}$ & None & $\begin{array}{l}\text { Mission replan, navigation to the } \\
\text { homing position and switch SAS to } \\
\text { EMERGENCY state }\end{array}$ \\
\hline
\end{tabular}

The information exchanged among the SAS, the high level $\mathrm{PC}$ and the remote control PC, is defined in four data frames which are described below:
- \$PFI2: data frame from the high level PC to the SAS, contains thrusters set points and command.

- SPFI3: data frame from the SAS to the high level PC, contains communications status, SAS and LIN nodes states, remaining energy and depth. This frame is published while the SAS is at the STANBY, REMOTE CONTROL STANDBY, ACTIVE or REMOTE CONTROL ACTIVE state.

- SPFI4: data frame from the SAS to the remote control PC vi through UHF radio link, contains communications status, GPS2 position data, remaining energy, depth and SAS and LIN nodes states. This frame is published while the SAS is at the REMOTE CONTROL STANDBY, REMOTE CONTROL ACTIVE, REMOTE CONTROL BEACON or BEACON state.

- \$PFI5: data frame from the emergency node via UHF radio link, contains GPS2 position data and SAS state. This frame is published while the SAS is at EMERGENCY state.

The data frames from the high level PC or remote control PC to the SAS contains a command field to modify the SAS operation state, as shown in Table II.

TABLE II. SAS COMMANDS

\begin{tabular}{|c|l|}
\hline Command & \multicolumn{1}{c|}{ Action } \\
\hline $\mathbf{0}$ & holds the SAS on STANBY state \\
\hline $\mathbf{1}$ & holds the SAS on ACTIVE state \\
\hline $\mathbf{5}$ & switch the SAS to BEACON state \\
\hline $\mathbf{7}$ & switch the SAS to EMERGENCY state \\
\hline
\end{tabular}

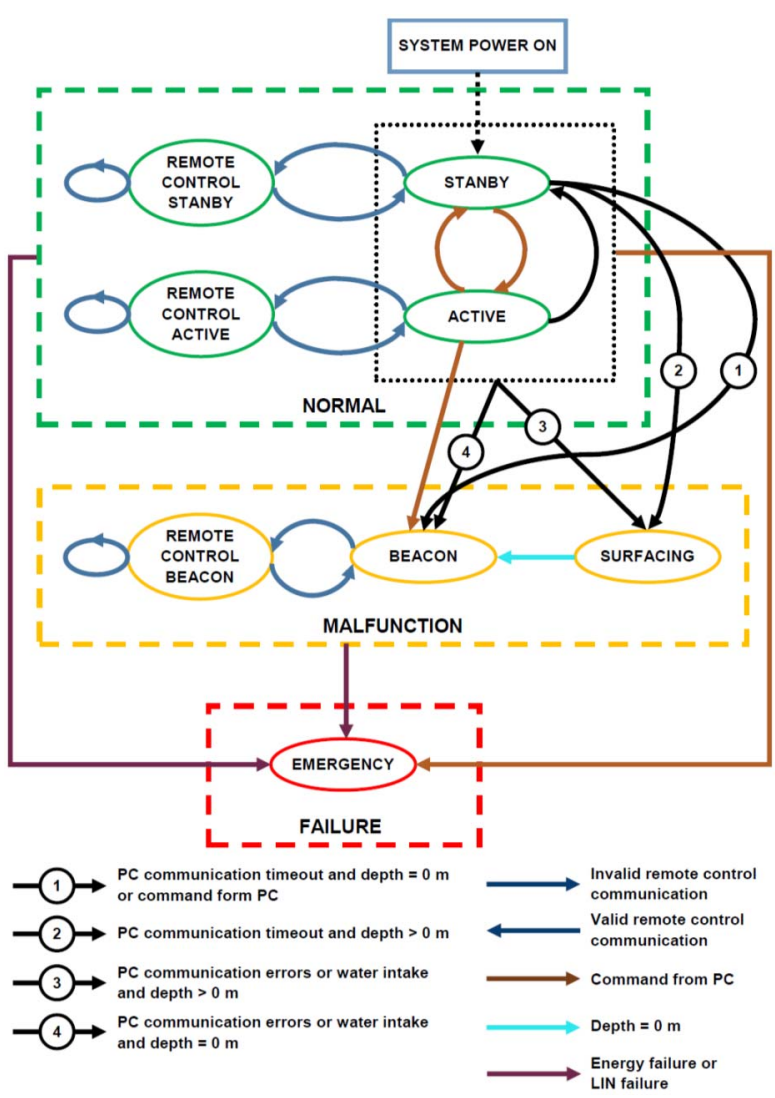

Fig. 5. Hub node sequential state machine states diagram. 
In Table III a brief description of the SAS operation states functionalities is provided.

TABLE III. SAS STATES AND META STATES

\begin{tabular}{|c|c|c|}
\hline & & Thrusters OFF \\
\hline & STANDBY & $\begin{array}{l}\text { SAS sends \$PFI3 every } 240 \mathrm{~ms} \\
\text { and waits for \$PFI2 }\end{array}$ \\
\hline & & $\begin{array}{l}\text { Emergency node holds the } \\
\text { ballast actuator. }\end{array}$ \\
\hline & & AUV in surface \\
\hline & CONTROL & $\begin{array}{l}\text { SAS receives \$PFI2 every } 1 \mathrm{~s} \\
\text { and sends } \$ P F I 3 \text { and } \$ P F I 4\end{array}$ \\
\hline NORMAL & & SAS holds the ballast actuator. \\
\hline & ACTIVE & $\begin{array}{l}\text { SAS sends \$PFI3 and waits for } \\
\text { \$PFI2 }\end{array}$ \\
\hline & ACIIVE & $\begin{array}{l}\text { Emergency node holds the } \\
\text { ballast actuator. }\end{array}$ \\
\hline & & AUV in surface \\
\hline & $\begin{array}{l}\text { REMOTE } \\
\text { CONTROL }\end{array}$ & $\begin{array}{l}\text { SAS receives \$PFI2 every 1s } \\
\text { and sends \$PFI3 and \$PFI4 }\end{array}$ \\
\hline & & $\begin{array}{l}\text { Emergency node holds the } \\
\text { ballast actuator. }\end{array}$ \\
\hline & & AUV submerged \\
\hline & SURFACING & $\begin{array}{l}\text { SAS shutdown the port and } \\
\text { starboard thrusters and set the } \\
\text { bow and stern thrusters speed } \\
\text { set points to emerge. }\end{array}$ \\
\hline & & $\begin{array}{l}\text { Emergency node releases the } \\
\text { ballast actuator. }\end{array}$ \\
\hline MU FUNCTIOY & & AUV in surface, thrusters OFF \\
\hline & BEACON & SAS sends $\$$ PFI4 \\
\hline & & $\begin{array}{l}\text { Emergency node holds the } \\
\text { ballast actuator. }\end{array}$ \\
\hline & & AUV in surface \\
\hline & $\begin{array}{l}\text { REMOTE } \\
\text { CONTROL }\end{array}$ & $\begin{array}{l}\text { SAS receives \$PFI2 every } 1 \mathrm{~s} \\
\text { and sends \$PFI4 }\end{array}$ \\
\hline & & $\begin{array}{l}\text { Emergency node holds the } \\
\text { ballast actuator. }\end{array}$ \\
\hline & & Thrusters OFF \\
\hline FAILURE & EMERGENCY & $\begin{array}{l}\text { Emergency node sends \$PFI5 } \\
\text { through the UHF transceiver. }\end{array}$ \\
\hline & & $\begin{array}{l}\text { Emergency node releases the } \\
\text { ballast actuator. }\end{array}$ \\
\hline
\end{tabular}

The high level electronics consists of an Advantech ${ }^{\circledR}$ ARK1122 PC with ROS built on top of the base Linux OS, the Xsens ${ }^{\circledR}$ MTI30-2A5G4 Inertial Measurement Unit (IMU), Garmin ${ }^{\circledR}$ 18xLVC GPS receiver (GPS1), Tritech ${ }^{\circledR}$ acoustic modem, Tritech $^{\circledR}$ Micron Forward Looking SONAR (FLS) and Tritech ${ }^{\circledR}$ Seaking Mechanical Scanning SONAR (MSS) these devices are connected to the PC via USB and serial ports. In addition, a WiFi router is connected to the PC via Ethernet to provide communication with others PCs.

In Fig. 6 two views of the Ictiobot-40 electronics chassis are shown.

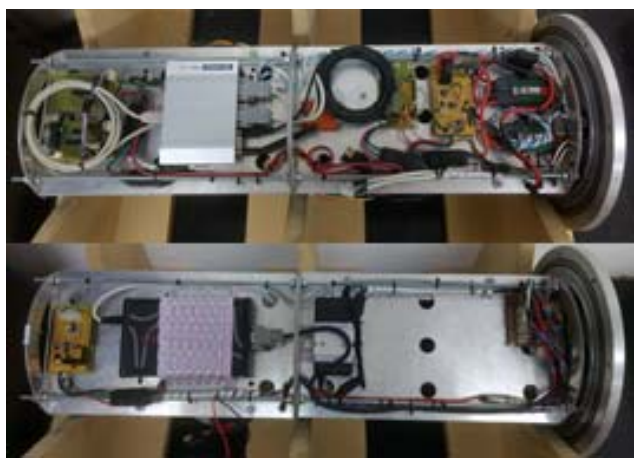

Fig. 6. Ictiobot-40 electronics chassis.

\section{PAYLOAD}

The AUV's payload consists of a Tritech ${ }^{\circledR}$ Seaking single beam Mechanical Scanning SONAR (MSS). This device is intended for obstacle detection in both AUVs and Remotely Operated Vehicles (ROV), but in this case it is used pointing downwards to detect seabed bottom. Given the relative low cost of this acoustic device against others for hydrographic applications, it is interesting to explore its potential use for bathymetric surveys. One disadvantage of this device for the intended application is the large beamwidth $\left(20^{\circ}\right)$ in the bowstern direction. Hence, further experimentation is still required to settle the impact of this beamwidth in this pretended application. In some scenarios this might be overcome through a mission plan that considers the navigation in a direction parallel to the maximum expected bottom slope.

The MSS is configured to have a vertical resolution of $0.075 \mathrm{~m}$ for ranges up to $60 \mathrm{~m}$. Longer ranges may be achieved, but the vertical resolution decreases. The swath angle is set to $60^{\circ}$ with a $0.45^{\circ}$ angle step.

\section{SOFTWARE DESIGN}

The Ictiobot-40 software architecture is based in the general framework previously introduced in Fig. 1, and developed with the Robot Operating System (ROS) [12] and [13]. The ROS implementation gave great advantages in terms of modularity, multicode compatibility and hardware abstraction. In addition, the use of rosbags files to register the navigation and payload data results very appropriate for post processing purposes.

Drivers for the SAS, MSS and FLS were written (SAS_ictiobot, MSS_driver, FLS_driver) in C++. Additionally, the Static Mission Plan, Dynamic Mission Planning, Navigation, Guidance, Control, Perception and Underwater Communications Systems (static_mission, DMPS ictiobot, NS_ictiobot, GS_ictiobot, CS_ictiobot, PS_ictiobot and UWAC ictiobot) packages were also programmed in different languages. This heterogeneity of programming languages encapsulated in the same software project is one of the outstanding features of ROS. The ROS nodes, which are the execution instance of the ROS packages, exchange data in messages form through the ROS topics.

These packages, nodes, services and topics diagram of the implemented architecture is presented in Fig. 7. 


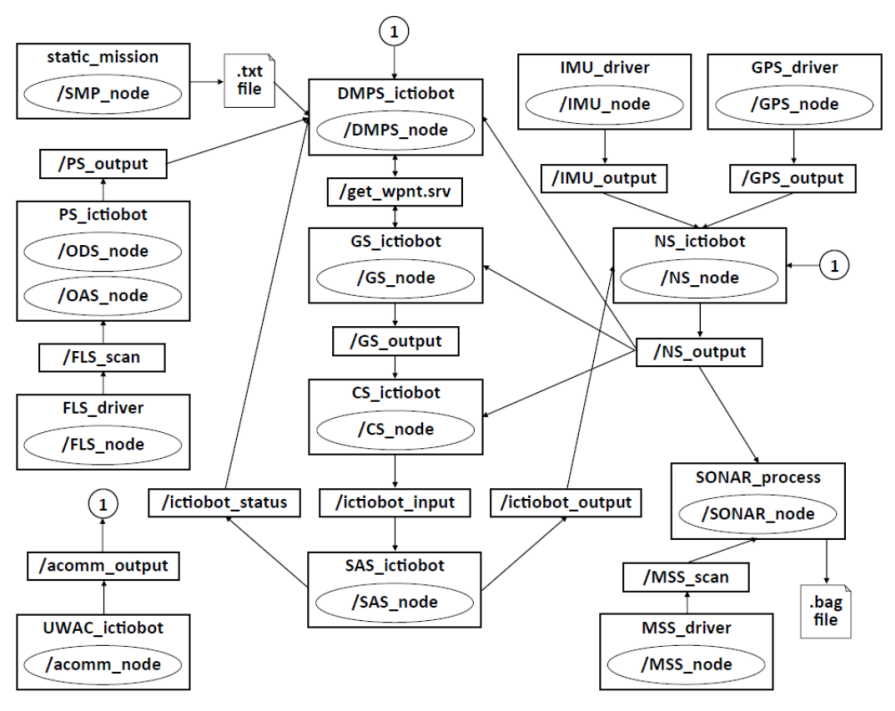

Fig. 7. Ictiobot- 40 software architecture.

\section{METHODOLOGY AND RESULTS}

In this section the methodology developed in the planning and execution of an autonomous acoustic survey with the Ictiobot-40 will be described. In addition, preliminary experimental results of the most relevant systems operating in wet tests are provided. However, the final full integration of all the AUV's systems has not yet been completed.

A typical mission for the Ictiobot-40 consists on an acoustic data survey. The objective of the mission is to reconstruct a bathymetric chart of the surveyed area using both MSS data applying bottom depth detection techniques and navigation system estimations of position and attitude. The mission will start at the deployment position (IWP, Initial WayPoint) from which the AUV moves to the survey area, where it describes a trajectory between the survey waypoints (SWP, Survey WayPoint) stored in the static mission plan while gathering acoustic and navigation data, once the survey area is covered, the AUV moves to a homing waypoint (HWP, Homing WayPoint), also stored in the static mission plan, where it is recovered after mission completion. During the development of the mission DMPS will introduce dynamic waypoints (DWP, Dynamic WayPoint) between the IWP, SWP and HWP, also the DMPS may introduce alternatives waypoints (AWP, Alternative WayPoint) if obstacles are detected in the current trajectory, further details of this will be given in the subsections bellow.

In order to implement this mission plan, a simple initial approach was chosen in which the Ictiobot-40 must describe a "lawn mower" trajectory in the survey area using Line Of Sight (LOS) guidance approach. The lawn mower trajectory will be planned to have a minimum overlap of the MSS data between legs and a surge velocity that allows obtaining a certain horizontal resolution of the MSS data. Both the overlap and surge velocity are dependents of the MSS configuration. A typical lawn mower trajectory is shown in Fig. 8, where the IWP, SWP, HWP, MSS swath coverage and overlap between trajectory legs are represented.
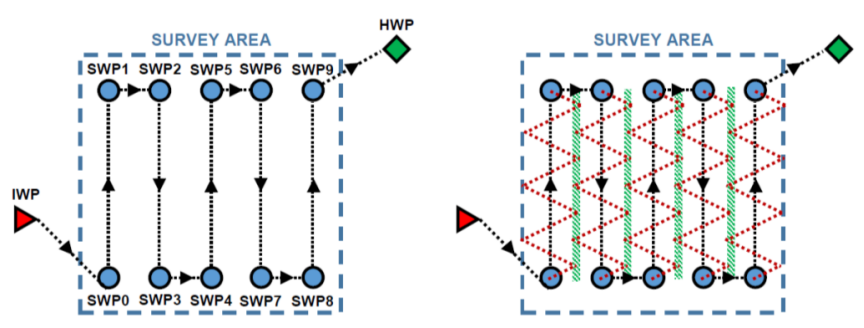

Fig. 8. Simplified mission scheme indentifying IWP, SWP and HWP (left) and MSS swath and overlap between legs (right).

\section{A. Static Mission Plan}

The static mission plan is determined by the survey waypoints SWPs, the surge velocity in the survey area and the homing position waypoint HWP. The SWPs are determined in terms of the latitude and longitude of the first SWP, length and width of the area of survey in meters, heading, expected MSS swath coverage in meters, and factor of overlapping between legs. This is programmed in the robot prior to the mission through its human-machine interface.

Then the SWPs of the lawn mower trajectory are obtained according to equations (1) and (2).

$$
\begin{gathered}
S W P_{-} L A T(k+1)=S W P_{-} L A T(k)+\left(\frac{\Delta Y}{R}\right) \\
S W P_{-} L N G(k+1)=S W P_{-} L N G(k)+\left[\frac{(\Delta X / R)}{\cos \left(S W P_{-} L A T(k)\right)}\right]
\end{gathered}
$$

Where; $S W P_{-} L A T(k+1)$ and $S W P_{-} L A T(k)$ are the latitudes of the $(k+1)$ and $(k)$ SWPs, $S W P_{-} L N G(k+1)$ and $S W P_{-} L N G(k)$ are the longitudes of the $(k+1)$ and $(k)$ SWPs, $R$ is the Earth radius (6378137 meters), $\Delta Y$ and $\Delta X$ are the SWPs displacements, in meters, in the North and East directions given by equations (3) and (4).

$$
\begin{gathered}
\Delta Y=-\operatorname{swath}(1-\text { overlap }) \cos \left(\frac{\pi}{2}-\psi\right) R L \\
\Delta X=\operatorname{swath}(1-\text { overlap }) \sin \left(\frac{\pi}{2}-\psi\right) R L
\end{gathered}
$$

Where; swath is the expected MSS swath coverage in meters, overlap is the overlapping factor, $\psi$ is the heading and $R L$ is a factor to determine is the survey will be done to the right $(R L=1)$ or to the left $(R L=-1)$.

The prior knowledge of the MSS swath coverage may be an issue in unexplored areas where the depth is unknown, in order to take that in account, and adaptive configuration of the MSS configuration parameters during the mission is considered as future work.

The surge velocity in the survey area will be set to $0.5 \mathrm{~m} / \mathrm{s}$ which allows to obtain an acceptable horizontal resolution.

The HWP is set by the user according to the most suitable position in which the AUV could be recovered.

In Fig. 9 an example of a static mission plan for a survey, including the IWP, lawn mower trajectory and HWP is shown. In this example, for the determination of the SWPs the; length, width, heading, expected MSS swath coverage and factor of 
overlapping were of; $200 \mathrm{~m}, 150 \mathrm{~m},-135^{\circ}, 10 \mathrm{~m}$ and 0.1 respectively.

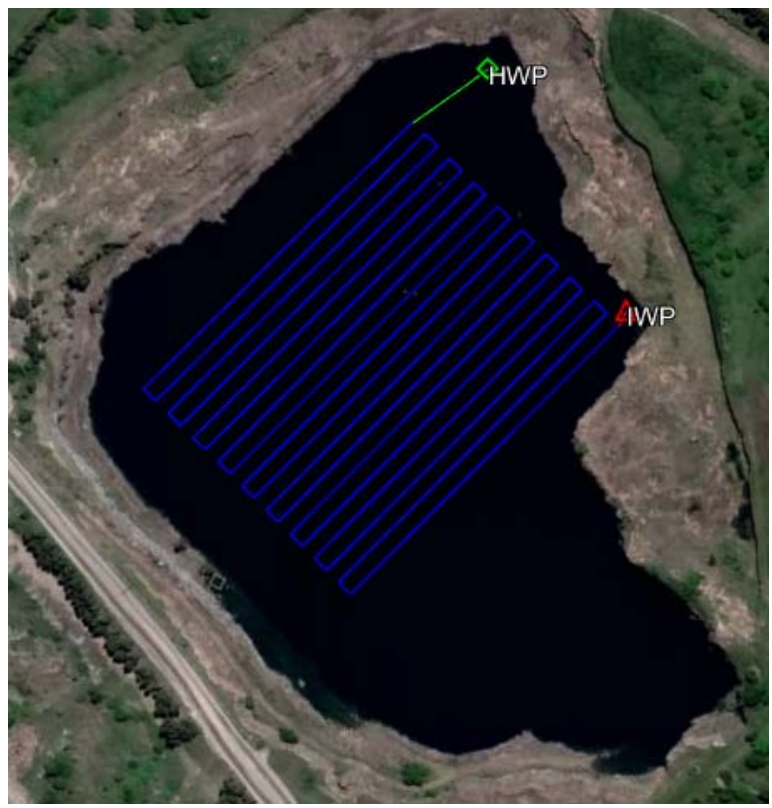

Fig. 9. Static Mission Plan generated for an example survey area.

\section{B. Dynamic Mission Planning System (DMPS)}

The DMPS, as mentioned before, generates short-term mission objectives. These objectives in the Ictiobot-40 respond to two basic circumstances: in normal operation, avoidance of obstacles in the planned trajectory and in case of failure or emergency, replan the mission objectives to preserve the integrity of the Ictiobot-40 [17].

To provide obstacle avoidance capabilities, the DMPS relies on data from the PS and NS. Based on this information the DMPS introduces two DWP between the actual SWP recently met by the AUV and the next SWP. In the absence of obstacles, these DWP are equally spaced in the trajectory between the two SWPs. If an obstacle is detected an AWP is introduced and its position is displaced from the normal trajectory allowing the AUV to navigate in an unobstructed direction. In Fig. 10 and 11 these situations are illustrated.
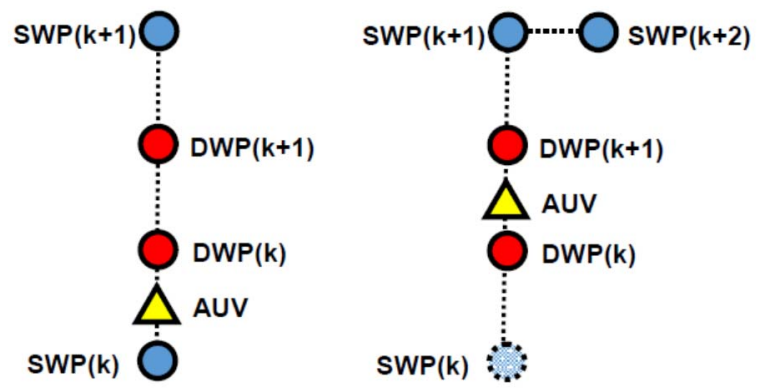

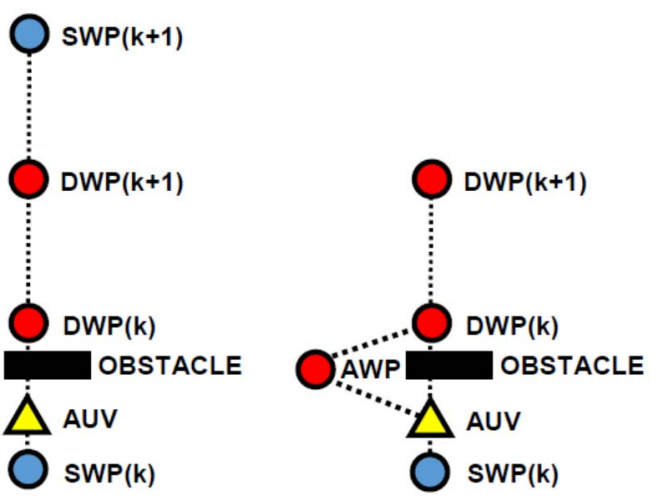

Fig. 11. Navigation on an obstructed trajectory.

In the case of failures or emergency, the DMPS take the actions mentioned in Table I or switches the SAS operation state using the commands listed in Table II.

\section{Navigation System (NS)}

The complete NS of Ictiobot-40 is under development. For surface navigation and to low depth where the GPS reception is still possible, the NS consists on a GPS aided Inertial Navigation System (GPS Aided INS) that uses measurements from an Xsens ${ }^{\circledR}$ IMU and a Garmin ${ }^{\circledR}$ GPS receiver. An Extended Kalman Filter (EKF) fusion algorithm is employed to determine velocity, position and attitude from the mentioned measurements [18].

Recently, the same system described before was improved by the implementation of a Fuzzy Logic adaptive EKF. In addition, an IMU calibration system is under test to reduce the systematic errors of the IMU inertial sensors measurements and hence, the growing rate of INS estimations errors. This IMU calibration system applies descending gradient and Particle Swarm Optimization (PSO) techniques to estimate the bias, scale factor and misalignment errors of IMU accelerometer, gyroscope and magnetometer sensor measurements.

For underwater navigation and positioning, where GPS reception is not available, range assistance and depth meter measurements will be used. The range from the AUV to a known reference position will be determined by the measurement of Time Of Flight (TOF) of acoustic communications between the AUV and an acoustic beacon in the reference position. This beacon might be a surface buoy or an Autonomous Surface Vehicle (ASV), such as the ASV Macábot, also developed by the INTELYMEC group [19].

In Fig. 12 a test trial of the GPS Aided INS of Ictiobot-40 showing position, North and East direction velocity and heading estimations is shown. This NS provides navigation data at a rate of $50 \mathrm{~Hz}$.

Fig. 10. Navigation on an unobstructed trajectory. 

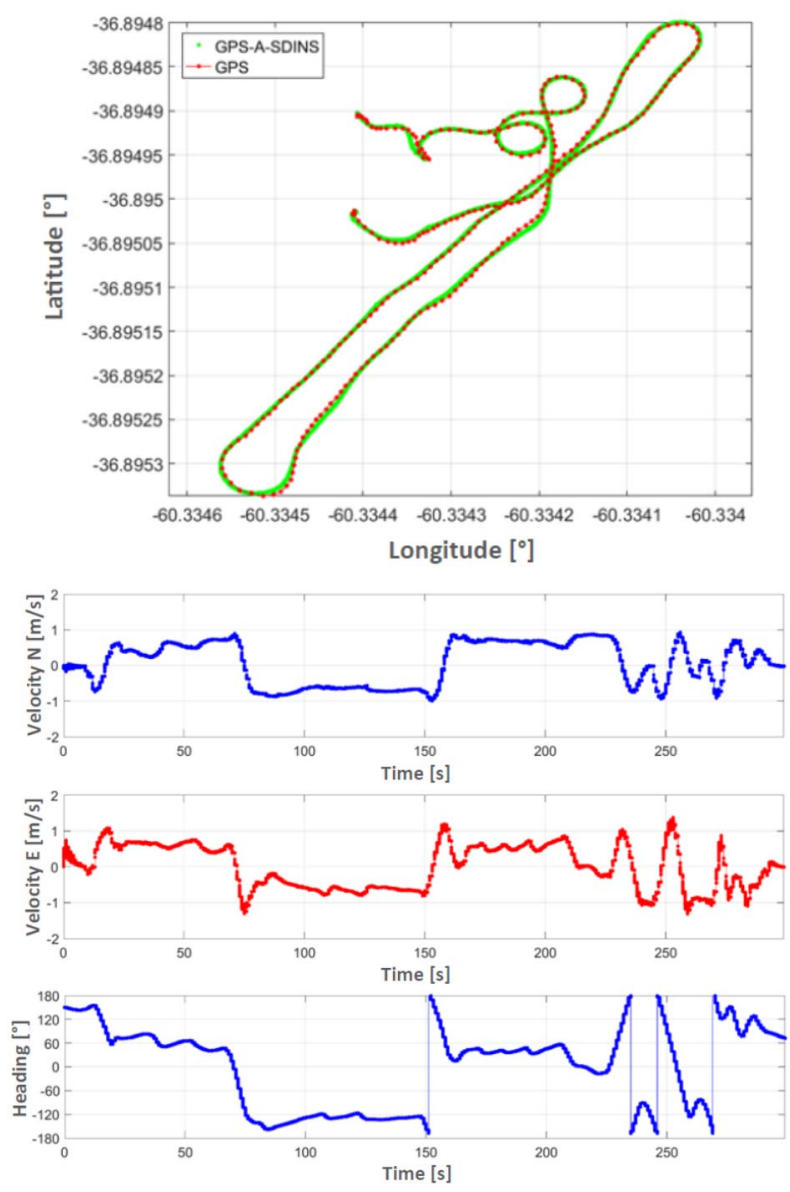

Fig. 12. Experimental results for position, $\mathrm{N}$ and $\mathrm{E}$ velocities and heading from the EKF based NS.

\section{Guidance System (GS)}

The Ictiobot-40 GS is based on the Line Of Sight (LOS) method. LOS provides a heading set point to the AUV course controller. This heading set point is obtained from the actual AUV position and the next waypoint position of the planned trajectory, as shown in equation (5).

$$
\psi_{W P}=\operatorname{atan} 2(a, b)
$$

Where, $a$ and $b$ are obtained with equations (6) and (7).

$$
\begin{gathered}
a=\sin \left(W P_{L N G}-A U V_{L N G}\right) \cos \left(W P_{L A T}\right) \\
b=\cos \left(A U V_{L A T}\right) \sin \left(W P_{L A T}\right) \\
-\sin \left(A U V_{L A T}\right) \cos \left(W P_{L A T}\right) \cos \left(W P_{L N G}-A U V_{L N G}\right)
\end{gathered}
$$

Where, $W P_{L A T}$ and $W P_{L N G}$ are the latitude and longitude of the next waypoint and $A U V_{L A T}$ and $A U V_{L N G}$ are the actual latitude and longitude of the AUV obtained from the NS data. All these variables are expressed in radians.

The AUV navigates to the next mission waypoint through the LOS guidance. Once the AUV position lies within a determined radius $R_{0}$ around the mission waypoint, satisfying next equation (8), this waypoint is considered as met, and the next waypoint in the trajectory is fed to the GS, as the new target waypoint.

$$
2 R|\operatorname{atan} 2(\sqrt{c}, \sqrt{1-c})| \leq R_{0}
$$

Where, $R$ is the Earth radius (6378137 m), $R_{0}$ is the circle of acceptance radius in meters and $c$ is obtained through equations (9), (10) and (11).

$$
\begin{gathered}
c=\sin ^{2}\left(\frac{\Delta L A T}{2}\right)+\cos \left(A U V_{L A T}\right) \cos \left(W P_{L A T}\right) \sin ^{2}\left(\frac{\Delta L N G}{2}\right) \\
\Delta L A T=\left(A U V_{L A T}-W P_{L A T}\right) \\
\Delta L N G=\left(A U V_{L N G}-W P_{L N G}\right)
\end{gathered}
$$

The DWPs introduced by the DMPS are beneficial to reduce the cross-track errors of the LOS guidance when the AUV must navigate to a waypoint located far away under the influence of perturbations such as currents and cross winds [20].

The LOS approach was selected for the initial developments due to its simplicity, however, in the future other trajectory tracking methods will be tested onboard of the Ictiobot-40, like [21] or Artificial Intelligence based techniques, like [22].

\section{E. Control System (CS)}

The Ictiobot-40 CS is based on three PID controllers for depth, heading and surge velocity. The feedback loops are closed using the information from the NS and the depth, heading and surge velocity setpoints given by the GS. The CS calculate the thrusters speed setpoints and from these the SAS generates the control signals for the thrusters drivers.

This CS scheme had been simulated using the dynamic model of Ictiobot-40 cited before [23]. Recently, other control schemes based in Artificial Intelligence techniques has been developed and will be tested in the near future [24] and [25].

\section{F. Perception System (PS)}

The PS of the Ictiobot-40 uses a Tritech ${ }^{\circledR}$ Micron mechanical scanning Forward Looking SONAR (FLS) pointed forward to detect the presence of obstacles in the AUV's trajectory. The PS consists in two subsystems, the Obstacle Detection System (ODS) and the Obstacle Avoidance System (OAS). The ODS processes the FLS data to detect and locate targets in every swath. Based on the ODS results, the OAS generates alternative waypoints (AWPs) to avoid the detected targets. These AWPs are fed to the DMPS that finally determines which of them is the most appropriate [26], [27] and [28]. In Fig. 14 and 15 an FLS echogram in a test tank and the resulting detections from the ODS are shown.

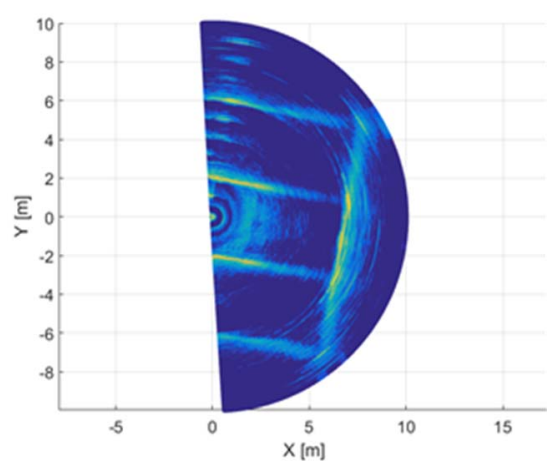

Fig. 13. Echogram for the FLS in a test tank, in cartesian coordinates. 


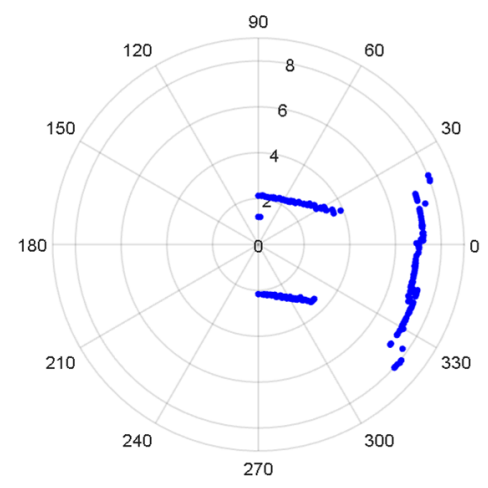

Fig. 14. Obstacles detections from the FLS data, in polar coordinates.

\section{G. Mechanical Scanning SONAR Processor (MSSP)}

In order to obtain a reconstruction of sea bottom images, and then perform threshold based bottom detection, the MSS data are processed to compensate absorption and geometrical spreading losses [29]. The depths of every MSS beam swath detection are corrected by the AUV attitude and position from the NS.

In a post processing stage the corrected gathered data are fed to the MBSystem software. An example of the resulting images from a test trial in a shallow water stream is shown in Fig. 15.

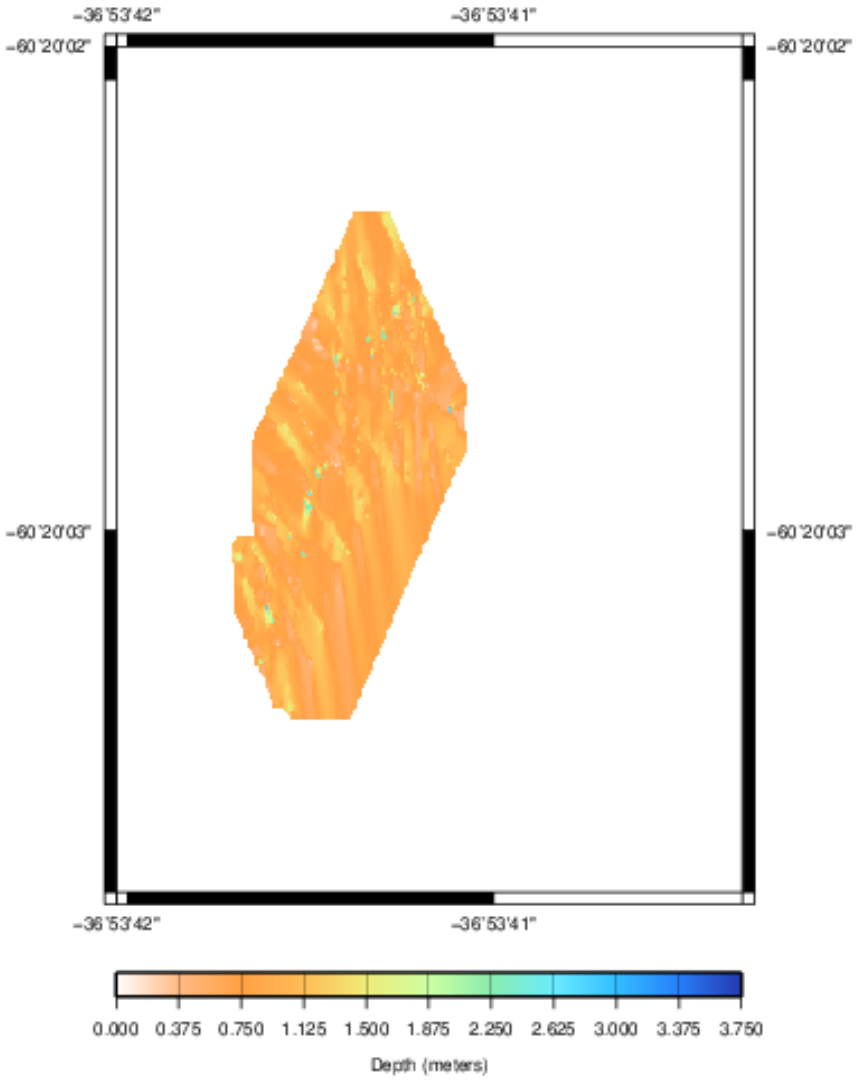

Fig. 15. Example of sea bottom image obtained from MSS and NS data.

\section{VIII.CONCLUSIONS}

From these preliminary test results it can be concluded that the Ictiobot-40 represents a great improvement with respect to the Ictiobot prototype.

The new electronic architecture with a well-defined boundary between the high and low level hierarchies, gives an adequate and practical level of abstraction to add several sensors and actuators without excessive $\mathrm{I} / \mathrm{O}$ ports requirements to the high level PC. Effectively, the SAS might be considered as another peripheral of the high level PC. The employment of the LIN in the SAS also allows achieving a highly modular, robust and scalable system.

In addition to this, the software architecture implementation in ROS proves to be very flexible, allowing multi language integration and hardware abstraction. It also provides a useful data gathering capability by the use of rosbags archives.

In the first test trials many of the developed systems of the Ictiobot-40 were tested with satisfactory results. This now constitutes a sound and reliable platform to keep on developing and testing new ideas.

Future improvements will consist of the incorporation of a Side Scan SONAR (SSS) to the PS; a Doppler Velocity Log (DVL) to the NS; and different approaches to the DMPS, the GS and the CS.

As a final remark, the Ictiobot-40 maintains the original easy to carry and low-cost premise.

\section{REFERENCES}

[1] C. von Alt, "Autonomous Underwater Vehicles," Woods Hole Oceanographic Institution, 2003.

[2] S. A. Gafurov, E. V. Klochkov, "Autonomous unmanned underwater vehicles development tendencies," Dynamics and Vibroacoustics of Machines, Procedia Engineering, vol. 106, pp. 141-148, 2015.

[3] S. Zongyuan, S. Junnan, M. Khushboo, and G. Shalabh, "An Autonomous Integrated System for 3-D Underwater Terrain Map Reconstruction," IEEE OCEANS 2016, Monterey, CA, 2016, pp. 1-6, 2016.

[4] S. Longfei, L. Yiping, Y. Shuxue, W. Jian, and C. Zhier, "Thermocline Tracking Using a Portable Autonomous Underwater Vehicle Based on Adaptive Threshold," IEEE OCEANS 2016, Shanghai, China, 2016, pp. $16-19$.

[5] M. Choyekh, N. Kato, R. Short, M. Ukita, H. Senga, M. Yoshie, E. Kobayashi, and H. Chiba, "Vertical Water Column Survey in the Gulf of Mexico Using Autonomous Underwater Vehicle SOTAB-I," Marine Technology Society Journal, vol. 49, pp. 88-101, 2015.

[6] H. Niu, S. Adams, T. Husain, N. Bose, and K. Lee, "Applications of Autonomous Underwater Vehicles in Offshore Petroleum Industry Environmental Effects Monitoring," Canadian International Petroleum Conference, 2007.

[7] S. Reed, Y. Petillot, and J. Bell, "Mine detection and classification in side scan sonar," Sea Technology., vol. 45, no. 11, pp. 35-39, 2004.

[8] S. Sariel, T. Balch, and N. Erdogan, "Naval Mine Countermeasure Missions," IEEE Robotics \& Automation Magazine, pp. 45-52, 2008.

[9] R. Dearden and J. Ernits, "Automated Fault Diagnosis for an Autonomous Underwater Vehicle," IEEE Journal of Oceanic Engineering, no. 3, vol. 38, 2013.

[10] O. Calvo, A. Sousa, J. Bibiloni, H. Curti, G. Acosta, and A. Rozenfeld, "Low-cost autonomous underwater vehicle for underwater acoustic inspections," Journal of Maritime Research., vol. 6, no. 2, pp. 37-52, 2009 . 
[11] G. Acosta, H. Curti, O. Calvo Ibañez, and S. Rossi, "Some Issues on the Design of a Low-Cost Autonomous Underwater Vehicle with an Intelligent Dynamic Mission Planner for Pipeline and Cable Tracking", Underwater Vehicles, IntechOpen, 2009.

[12] M. Quigley, K. Conley, B. P. Gerkey, J. Faust, T. Foote, J. Leibs, R. Wheeler, A. Y. Ng, "ROS: an open-source Robot Operating System," ICRA Workshop on Open Source Software. 2009.

[13] A. Koubaa, Robot Operating System (ROS) The Complete Reference, 1st. Ed., vol. 1. Springer International Publishing, 2016.

[14] A. Petit, C. Paulo, I. Carlucho, B. Menna, and M. D. Paula, "Prediction of the hydrodynamic coefficients of an autonomous underwater vehicle," 2016 3rd IEEE/OES South American International Symposium on Oceanic Engineering (SAISOE), 2016, pp. 1-6.

[15] S. C. Talbot, and S. Ren, "Comparison of FieldBus Systems, CAN, TTCAN, FlexRay and LIN in Passenger Vehicles," 29th IEEE International Conference on Distributed Computing Systems Workshops, 2009.

[16] LIN - Local Interconnect Network, "LIN Specification 2.2A," www.linsubbus.org, 2013

[17] G. G. Acosta, H. Curti, O. Calvo, and S. Rossi, "A Knowledge-based approach for an AUV Path Planner Development," WSEAS Transactions on Systems, Hangzhou, 2006, vol. 5 p. 1417 - 1424.

[18] B. V. Menna, S. A. Villar, A. Rozenfeld, and G. G. Acosta, "GPS aided strapdown inertial navigation system for autonomous robotics applications," 2017 XVII Workshop on Information Processing and Control (RPIC), 2017, pp. 1-6.

[19] G. G. Acosta, B. V. Menna, R. J. de la Vega, L. M. Arrien, H. J. Curti, S. A. Villar, R. C. Leegstra, M. De Paula, I. Carlucho, F. J. Solari, and A. F. Rozenfled, "MACÁBOT: prototipo de vehículo autónomo de superficie (ASV)," 2017 IX Jornadas Argentinas de Robótica (JAR), 2017, pp. 1-6.

[20] T. I. Fossen, Marine Control Systems Guidance, Navigation, and Control of Ships, Rigs and Underwater Vehicles. Trondheim, Norway, Marine Cybernetics, 2002.

[21] A. F. Rozenfled, G. G. Acosta, A. Sousa, H. J. Curti, and O. A. Calvo, “A guidance and control system proposal for autonomous pipeline inspections," Transactions on Systems, Signals \& Devices, Aachen, 2010, vol. 5, pp. $5-27$.

[22] M. De Paula and G. G. Acosta, "Trajectory tracking algorithm for autonomous vehicles using adaptive reinforcement learning," OCEANS 2015 - MTS/IEEE Washington, 2016.

[23] I. Carlucho, B. V. Menna, M. D. Paula, and G. G. Acosta, "Comparison of a PID controller versus a LQG controller for an autonomous underwater vehicle," 2016 3rd IEEE/OES South American International Symposium on Oceanic Engineering (SAISOE), 2016, pp. 1-6.

[24] I. Carlucho, M. De Paula, W. Sen, Y. Petillot, and G. G. Acosta, "Adaptive low-level control of autonomous underwater vehicles using deep reinforcement learning," Robotics and Autonomous Systems, 2018, vol. 107 , p. $71-86$.

[25] I. Carlucho, M. De Paula, S. A. Villar, and G. G. Acosta, "Incremental Qlearning strategy for adaptive PID control of mobile robots," Expert Systems with Applications, 2017, vol. 80, p. 183 - 199.

[26] S. A. Villar, F. J. Solari, B. V. Menna, and G. G. Acosta, "Obstacle detection system design for an autonomous surface vehicle using a mechanical scanning sonar," 2017 XVII Workshop on Information Processing and Control (RPIC), 2017, pp. 1-6.

[27] S. A. Villar, G. G. Acosta, A. Sousa, A. F. Rozenfeld, "Evaluation of an efficient approach for target tracking from acoustic imagery for the perception system of an autonomous underwater vehicle," International Journal of Advanced Robotic Systems, 2014.

[28] F. J. Solari, A. F. Rozenfeld, S. A. Villar and G. G. Acosta, "Artificial potential fields for the obstacles avoidance system of an AUV using a mechanical scanning sonar," 2016 3rd IEEE/OES South American International Symposium on Oceanic Engineering (SAISOE), Buenos Aires, 2016, pp. 1-6.

[29] R. J. Urick, Principles of Underwater Sound. Westport, CT, USA, Peninsula Publishing, 1996. 